\title{
Thermal Conductivity and Tensile Properties of Carbon Nanofiber- Reinforced Aluminum-Matrix Composites Fabricated via Powder Metallurgy: Effects of Ball Milling and Extrusion Conditions on Microstructures and Resultant Composite Properties
}

\author{
Fumio Ogawa $^{1} \cdot$ Shuji Yamamoto ${ }^{2} \cdot$ Chitoshi Masuda $^{3}$
}

Received: 14 May 2018/Revised: 20 June 2018/Published online: 5 September 2018

(C) The Chinese Society for Metals and Springer-Verlag GmbH Germany, part of Springer Nature 2018

\begin{abstract}
Carbon nanofiber (CNF)-reinforced aluminum-matrix composites were fabricated via ball milling and spark plasma sintering (SPS), SPS followed by hot extrusion and powder extrusion. Two mixing conditions of CNF and aluminum powder were adopted: milling at $90 \mathrm{rpm}$ and milling at $200 \mathrm{rpm}$. After milling at $90 \mathrm{rpm}$, the mixed powder was sintered using SPS at $560{ }^{\circ} \mathrm{C}$. The composite was then extruded at $500{ }^{\circ} \mathrm{C}$ at an extrusion ratio of 9 . Composites were also fabricated via powder extrusion of powder milled at $200 \mathrm{rpm}$ and $550{ }^{\circ} \mathrm{C}$ with an extrusion ratio of 9 (R9) or 16 (R16). The thermal conductivity and tensile properties of the resultant composites were evaluated. Anisotropic thermal conductivity was observed even in the sintered products. The anisotropy could be controlled via hot extrusion. The thermal conductivity of composites fabricated via powder extrusion was higher than those fabricated using other methods. However, in the case of specimens with a CNF volume fraction of $4.0 \%$, the thermal conductivity of the composite fabricated via SPS and hot extrusion was the highest. The highest thermal conductivity of $4.0 \% \mathrm{CNF}$-reinforced composite is attributable to networking and percolation of CNFs. The effect of the fabrication route on the tensile strength and ductility was also investigated. Tensile strengths of the R9 composites were the highest. By contrast, the R16 composites prepared under long heating duration exhibited high ductility at CNF volume fractions of $2.0 \%$ and $5.0 \%$. The microstructures of composites and fracture surfaces were observed in detail, and fracture process was elucidated. The results revealed that controlling the heating and plastic deformation during extrusion will yield strong and ductile composites.
\end{abstract}

Keywords Al composite $\cdot$ Carbon nanofiber $\cdot$ Ball milling $\cdot$ Hot extrusion $\cdot$ Microstructure and performance

\section{Introduction}

Carbon nanotubes (CNTs) are expected to function as an ideal reinforcement for composite materials because of the lightweight, high strength, high thermal conductivity, and

Available online at http://link.springer.com/journal/40195

Fumio Ogawa

ogawa-f@fc.ritsumei.ac.jp

Shuji Yamamoto

shujiy32@gmail.com

Chitoshi Masuda

masuda-chitoshi@waseda.jp

1 Department of Mechanical Engineering, College of Science and Engineering, Ritsumeikan University, 1-1-1, Nojihigashi, Kusatsu-shi, Shiga 525-8577, Japan good electrical conductivity [1-3]. CNT-reinforced polymer-matrix composites have been widely studied, and composites used in actual engineering applications have been developed [4]. Compared with nano-reinforced polymer-matrix composites, CNT-reinforced metal-matrix

2 Kanto Gakuin University, 1162-2, Ogikubo, Odawara-shi, Kanagawa 250-0042, Japan

3 Kagami Memorial Institute for Materials Science and Technology, Waseda University, 2-8-26, Nishi-Waseda, Shinjuku-ku, Tokyo 169-0051, Japan 
composites have not been extensively studied [5-7]. CNTreinforced aluminum-matrix composites which have potential applications in automobiles, aerospace vehicles, and thermal management devices [8-26] have attracted increased research attention.

Powder metallurgy can circumvent the poor wettability of CNTs by molten aluminum and is widely used for in fabricating composites. The degrees of dispersion and the fracture of CNTs can be controlled during ball milling by ball milling speed and milling duration, and CNTs can be aligned via hot extrusion. To date, grain refinement via ball milling and the alignment of CNTs are effective approaches for improving the strength of CNT-reinforced aluminum-matrix composites $[11,24,25]$. However, the mechanism responsible for the thermal conductivity of such composites has not been elucidated. Shin et al. [20] and $\mathrm{Wu}$ et al. [21] discussed the thermal conductivity of CNT-reinforced aluminum-matrix composites in detail. Microstructures, such as dispersion state and orientation of CNTs, presumably affect the thermal conductivity of composites; however, few studies have verified the effect on the CNT-reinforced aluminum-matrix composites.

In this study, aluminum-matrix composites reinforced with carbon nanofibers (CNFs), a type of CNTs with $150 \mathrm{~nm}$ diameter, were fabricated via ball milling, spark plasma sintering (SPS), and hot extrusion. Two ball milling speeds and two durations were adopted to investigate the effects of the degree of dispersion of CNTs and fracture on the properties of the resultant composites. Composites were fabricated via three fabrication routes: SPS, SPS followed by hot extrusion, and powder extrusion [24, 25]. In powder extrusion, a mixed powder of CNFs and aluminum was encapsulated into an aluminum container and extruded at $550{ }^{\circ} \mathrm{C}$. The thermal conductivity of the obtained composites was subsequently evaluated. In particular, specimens after SPS and SPS followed by hot extrusion were cut, and the thermal conductivity in the direction parallel to and perpendicular to the press or extrusion direction was evaluated. The observed anisotropy in thermal conductivity was discussed. The tensile properties of the composites were also investigated. The effects of ball milling speed, heating duration before extrusion, and extrusion ratio [9 (R9) or 16 (R16)] on the tensile strength, ductility, and fracture process of the composites were investigated. Some fabrication conditions to achieve these tensile properties were selected based on previous studies [24, 25]. In a previous study, composites with unprecedently superior mechanical properties and thermal conductivity were fabricated by optimizing the mixing conditions of CNFs and aluminum to homogeneously disperse CNFs and to avoid extensively fracturing CNFs during milling [25]. In the study, the CNFs and aluminum powder were mixed in a planetary ball mill (Fritsch Pulverisette 5). Stainless jars containing CNFs, aluminum powder, stainless-steel balls, and stearic acid $(\mathrm{C} 17 \mathrm{H} 35 \mathrm{COOH})$ were rotated at $200 \mathrm{rpm}$ for $3 \mathrm{~h}$. Weight fraction of stainless balls (relative to the CNT-aluminum powder mixture) was 20:1. However, the fabrication of composites via SPS, SPS followed by hot extrusion, and powder extrusion with R16 has not been reported yet. Herein, the variation in microstructures, such as aluminum grains and CNF orientation, due to fabrication conditions was analyzed. The fracture mechanism was investigated by observing the fracture surface of the composites. The relation between the fabrication conditions and composite properties as well as the mechanism governing this relation was discussed.

\section{Experimental}

Aluminum powder and CNFs (VGCF; Showa Denko Co. Ltd., Japan; average diameter: $150 \mathrm{~nm}$, average length: $15 \mu \mathrm{m}$ ) were mixed in a planetary ball mill (Fritsch Pulverisette 5). Figure 1 shows the SEM image of CNFs, revealing CNFs' fiber-like structure. The ratio of the intensity of $\mathrm{D}$ band $\left(1360 \mathrm{~cm}^{-1}\right)$ to that of $\mathrm{G}$ band $\left(1580 \mathrm{~cm}^{-1}\right)$, i.e., $I_{\mathrm{D}} / I_{\mathrm{G}}$, was calculated from the Raman spectra to be 0.071 , which indicates the high purity of CNFs.

Two methods were used for ball milling. The first was mild milling with a rotation speed of $90 \mathrm{rpm}$ and a milling duration of $3 \mathrm{~h}$. The average size of aluminum powder particles used was $3 \mu \mathrm{m}$. Stainless balls were used for milling without a process-control agent, and the ball-toweight ratio was 20:1. Mixed powder was embedded in a commercial epoxy resin in order to observe its interior. The resins were polished, and their surfaces were etched using

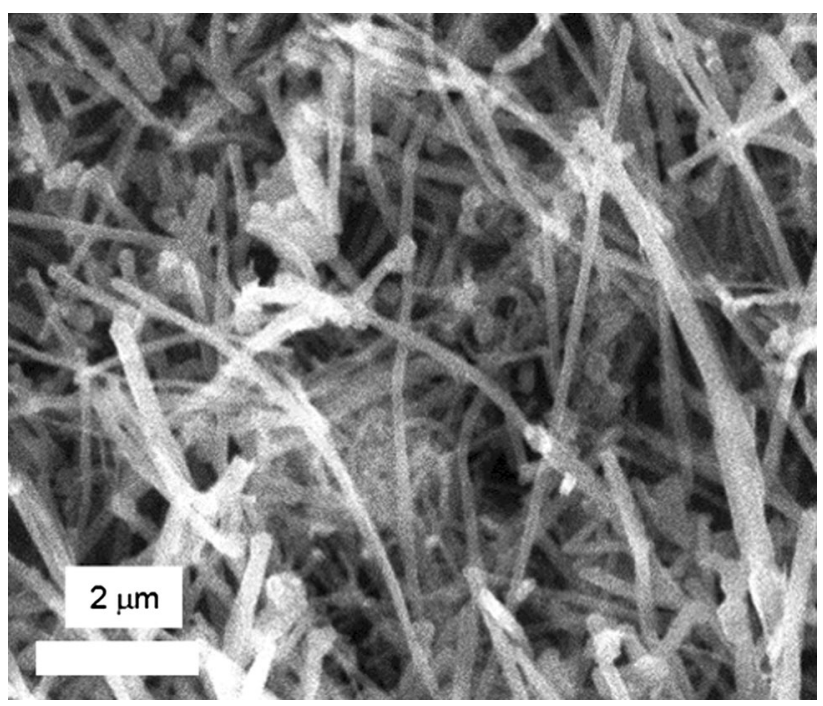

Fig. 1 SEM image of CNFs 


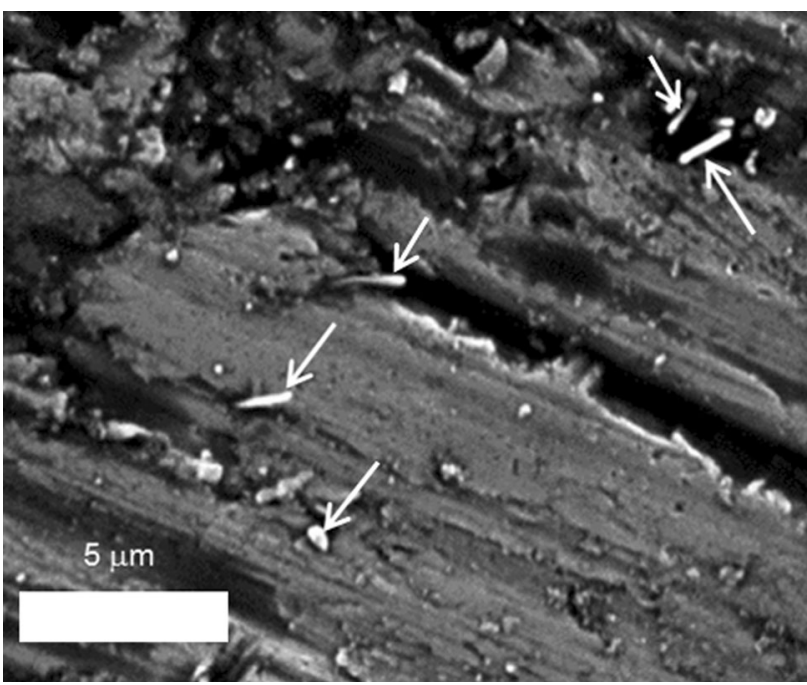

Fig. 2 SEM image of inside mixed powder of CNFs and aluminum with $\mathrm{CNF}$ volume fraction of $1.0 \%$ after ball milling at $90 \mathrm{rpm}$

nitric acid in order to study the distribution of CNFs inside the powders. Figure 2 shows the SEM image of inside mixed powder. CNFs are indicated by white arrows, indicating its almost homogeneous dispersion in aluminum powder particles.

The second milling was performed at $200 \mathrm{rpm}$ for $3 \mathrm{~h}$. The average size of the aluminum powder particles was $30 \mu \mathrm{m}$. Stearic acid $(1.5 \mathrm{wt} \%)$ was used as a processcontrol agent, and the ball-to-weight ratio was 20:1. To prevent excessive cold welding of the powder, rotation for $20 \mathrm{~min}$ followed by suspension for $40 \mathrm{~min}$ was repeated nine times [24]. The average length of CNFs after milling was $6 \mu \mathrm{m}$ [24]. The mixed powder after mild milling was sintered via SPS at $560{ }^{\circ} \mathrm{C}$ for $2 \mathrm{~h}$ under $50 \mathrm{MPa}$ using a sintering machine, a Dr. Sinter system (SPS Syntex), thereby yielding a sintered product with $30 \mathrm{~mm}$ diameter and $20 \mathrm{~mm}$ height. Under these sintering conditions, dense composites were obtained and no peaks of aluminum carbide $\left(\mathrm{Al}_{4} \mathrm{C}_{3}\right)$, the reactant of aluminum and $\mathrm{CNFs}$, were evident in the X-ray diffraction (XRD) patterns of the products (data omitted here). Hot extrusion of the sintered product was performed using a conical die at $\mathrm{R} 9$, extrusion temperature of $500{ }^{\circ} \mathrm{C}$, and extrusion speed of $0.1 \mathrm{~mm} /$ min. Extrusion was performed immediately after the temperature reached $500{ }^{\circ} \mathrm{C}$.

Pieces with a dimension of $10.0 \mathrm{~mm} \times 10.0 \mathrm{~mm} \times$ $3.0 \mathrm{~mm}$ were cut from the sintered products or the extruded rod (Fig. 3). " $T$ " denotes the plane transverse to the press or extrusion direction and " $P$ " denotes the plane parallel to the press or extrusion direction. Thermal conductivities in the sintering press or extrusion direction and in the respective perpendicular directions were measured using the laser flash method (NETZSCH; LFA447
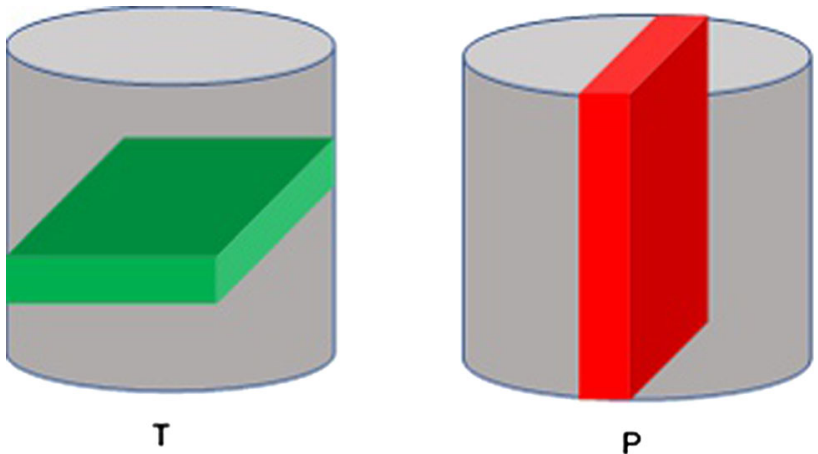

Fig. 3 Planes for thermal conductivity measurements

NanoFlash) to investigate the effect of pressing and hot extrusion. Three measurements were performed for each specimen.

Tensile properties were also evaluated for extruded composites. The length of the tensile specimen was $50 \mathrm{~mm}$, whereas its thickness and gage length were 2 and $13 \mathrm{~mm}$, respectively [26]. Tensile tests were performed using a tensile testing machine (Shimadzu; AG-100KNC) at a cross-head speed of $0.5 \mathrm{~mm} / \mathrm{min}$. Strain was monitored using strain gages attached to the center of the gage section.

Composites were also fabricated via powder extrusion. The mixed powder after milling at a rotation speed of $200 \mathrm{rpm}$ was encapsulated into an aluminum container. The powders were compressed under vacuum $\left(10^{-5}\right.$ Torr $)$ using the SPS system (Dr. Sinter; SPS Syntex) and capped with an aluminum lid applied under force. The SPS system was used only for creating the vacuum, compressing the powders, and installing the lid. Pore formation and oxidation of the compressed powder were carefully avoided during heating in the hot extrusion process. Residual air in the container can oxidize the powder. The containers were then machined into extrusion billets of $30 \mathrm{~mm}$ diameter and $45 \mathrm{~mm}$ height. Hot extrusion was performed using a conical die with an angle of $60^{\circ}$ at R9, extrusion temperature of $550{ }^{\circ} \mathrm{C}$, and extrusion speed of $20 \mathrm{~mm} / \mathrm{min}$. Extrusion was performed after the temperature was maintained at $550{ }^{\circ} \mathrm{C}$ for $0.5 \mathrm{~h}$. Powder extrusion at R16 was also performed. An extrusion billet of $40 \mathrm{~mm}$ diameter and height, in which the mixed powder was encapsulated, was prepared. Extrusion was performed at $550{ }^{\circ} \mathrm{C}$ using conical die with an angle of $60^{\circ}$. Extrusion was performed after the temperature was maintained at $550{ }^{\circ} \mathrm{C}$ for $1.5 \mathrm{~h}$ to avoid cracking in the extruded materials; the extrusion speed was $20 \mathrm{~mm} / \mathrm{min}$. Table 1 summarizes the fabrication conditions of composites in this study.

The thermal conductivity of the composites fabricated via powder extrusion with $\mathrm{R} 9$ in the extrusion direction was evaluated using the laser flash method (NETZSCH; 
Table 1 Fabrication conditions of CNF-reinforced aluminum-matrix composites

\begin{tabular}{lcc}
\hline Fabrication route & Ball-milling conditions & SPS and/or hot extrusion condition \\
\hline SPS & $90 \mathrm{rpm}, 3 \mathrm{~h}$, ball-to-weight ratio $=20: 1$ & SPS: $560{ }^{\circ} \mathrm{C}, 2 \mathrm{~h}, 50 \mathrm{MPa}$ \\
& (aluminum particle size: $3 \mu \mathrm{m})$ & \\
SPS and hot extrusion & $90 \mathrm{rpm}, 3 \mathrm{~h}$, ball-to-weight ratio $=20: 1$ & SPS: $560{ }^{\circ} \mathrm{C}, 2 \mathrm{~h}, 50 \mathrm{MPa}$ Hot extrusion: $500{ }^{\circ} \mathrm{C}$, \\
& (aluminum particle size: $3 \mu \mathrm{m})$ & $0.1 \mathrm{~mm} / \mathrm{min}, \mathrm{R} 9$ \\
Powder extrusion & $200 \mathrm{rpm}, 3 \mathrm{~h}$, ball-to-weight ratio $=20: 1$ & Powder extrusion: $550{ }^{\circ} \mathrm{C}, 20 \mathrm{~mm} / \mathrm{min}, \mathrm{R} 9(\mathrm{holding}$ \\
& (aluminum particle size: $30 \mu \mathrm{m})$ & time: $0.5 \mathrm{~h}), \mathrm{R} 16($ holding time: $1.5 \mathrm{~h})$ \\
\hline
\end{tabular}

LFA447 NanoFlash). Three measurements were performed for each specimen. Tensile tests were performed for composites fabricated via powder extrusion with R9 and R16. Tensile tests were performed at a cross-head speed of $0.5 \mathrm{~mm} / \mathrm{min}$ using Shimadzu AG-100KNC. The specimens were designed according to Japan Industrial Standard (JIS) $\mathrm{Z}$ 2241. Strain was measured using high-elongation strain gages attached at the center of the gage section.

\section{Results and Discussion}

\subsection{Thermal Conductivity}

Figure 4 shows the thermal conductivity measurement results for the SPS composites. A large difference is observed in the thermal conductivities of the specimens in the $T$ and $P$ directions. Compared with the thermal conductivity perpendicular to the $\mathrm{P}$ plane in the aluminum, the thermal conductivity in specimens with $\mathrm{CNF}$ volume fractions of $0.5 \%$ and $1.0 \%$ increased. At a CNF volume fraction of $1.0 \%$, the thermal conductivity of aluminum is higher than that of bulk aluminum $(237 \mathrm{~W} / \mathrm{m} \mathrm{K})$. Wu et al. [21] reported the highest thermal conductivity of $199 \mathrm{~W} /$ $\mathrm{m} \mathrm{K}$ for $0.5 \mathrm{wt} \% \mathrm{CNT}$-reinforced aluminum composites. The values obtained herein are quite high compared with

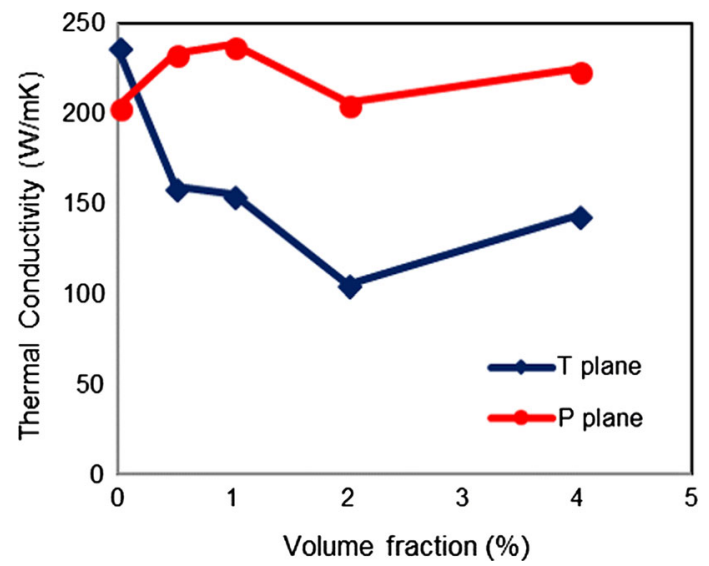

Fig. 4 Thermal conductivities of the sintered CNF-aluminum composites in the directions perpendicular to the $T$ and $P$ planes as a function of the CNF volume fraction those reported previously [20, 21]. Thermal conductivities perpendicular to the $T$ plane decrease with increasing $\mathrm{CNF}$ volume fraction. Anisotropy in the thermal conductivities is observed even in the sintered products probably owing to the orientation of CNFs in composites. The thermal conductivities decrease as the CNF volume fraction increases to $2.0 \%$. However, the conductivities continue to increase at a volume fraction of $3.0 \%$. In the study of $\mathrm{Wu}$ et al. [21], thermal conductivity monotonically decreased with increasing fraction of CNTs. However, herein, thermal conductivity increases again at $3.0 \%$ due to the networking and percolation of CNFs because of mild dispersion condition of CNFs.

Figure 5 shows the SEM images of the $T$ and $P$ planes of sintered products with $1.0 \%$ CNFs. For observation, surfaces were polished and etched using nitric acid solution. In Fig. 5, the CNFs are indicated by white arrows. Apparently, CNFs did not experience fracture during milling; thus, no peaks of $\mathrm{Al}_{4} \mathrm{C}_{3}$ are detected by XRD. CNFs are clearly oriented perpendicular to the press direction in the $T$ plane of the sintered product, whereas those in the $P$ plane are not perpendicularly oriented. The orientation of the CNFs affected the thermal conductivities of the sintered composites.

Figure 6 shows the thermal conductivities of composites fabricated via SPS followed by hot extrusion. The thermal conductivities perpendicular to the $T$ plane after hot extrusion increase compared with those perpendicular to the $T$ plane of the sintered products, whereas the thermal conductivities perpendicular to the $P$ plane decrease after hot extrusion compared with those perpendicular to the $P$ plane of the sintered products. At CNF contents of $0.5 \%$ and $1.0 \%$, the thermal conductivities perpendicular to the $T$ plane are higher than those perpendicular to the $P$ plane of the extruded composites. Thus, hot extrusion effectively improves the thermal conductivities of the sintered specimens, enabling to control the degree of the thermal conductivity. To our knowledge, the control of thermal conductivity due to hot extrusion and/or rolling has not been reported yet [21, 22].

Figure 7 shows the XRD patterns on the $P$ planes of the extruded rod and sintered product with $4.0 \% \mathrm{CNF}$ content. The pattern of the extrudate shows a peak at $26.4^{\circ}$ 

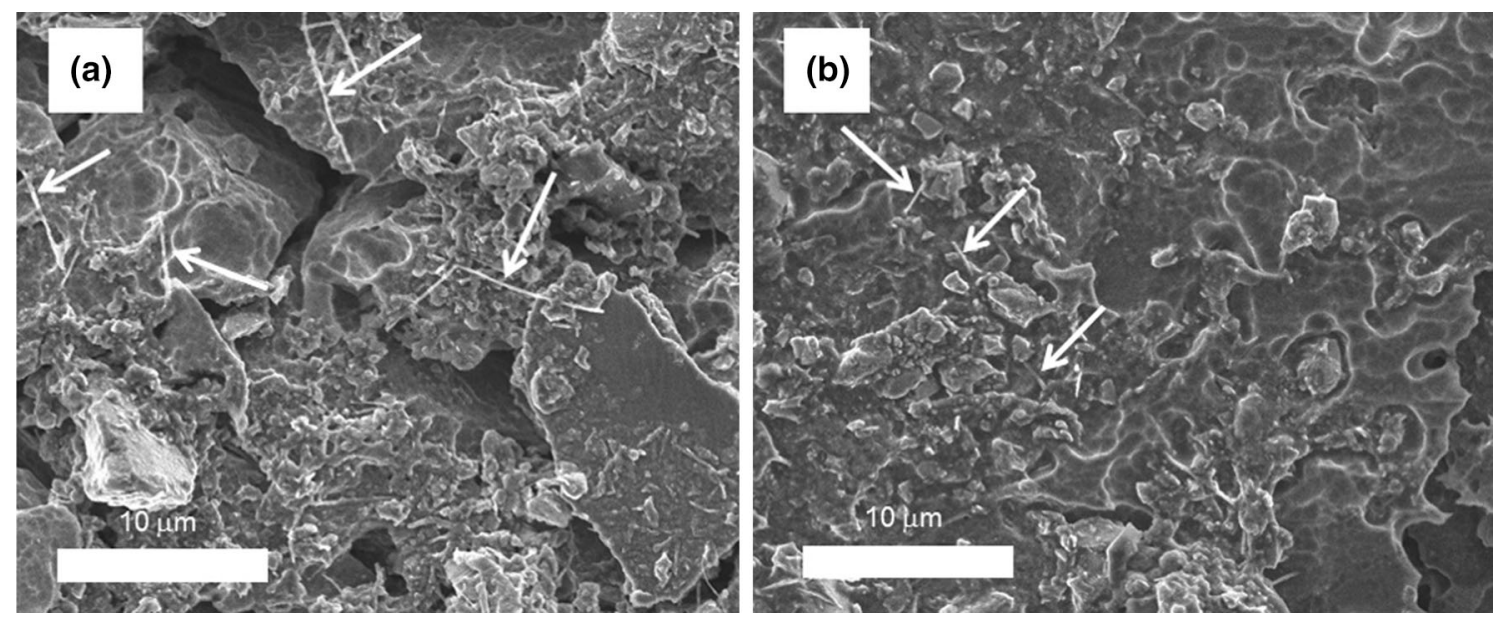

Fig. 5 SEM images of the sintered $1.0 \%$ CNF-reinforced composites: a $T$ plane, $\mathbf{b} P$ plane

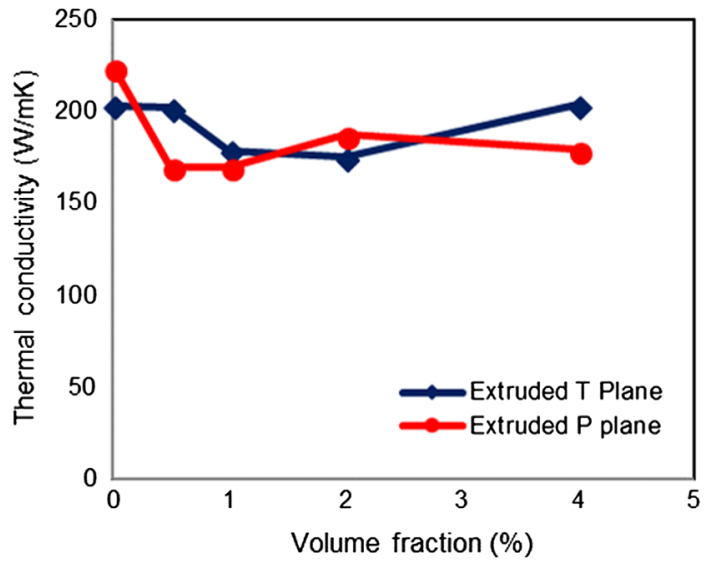

Fig. 6 Thermal conductivities of CNF-aluminum composites fabricated via SPS followed by hot extrusion, plotted as a function of the $\mathrm{CNF}$ volume fraction

corresponding to the ( $\left(\begin{array}{lll}0 & 0\end{array}\right)$ plane of the CNFs; by contrast, no such peak is evident in the sintered product. The CNFs are apparently aligned with the extrusion direction; such an alignment is responsible for the observed improvement in the thermal conductivity after hot extrusion.

Figure 8 shows an SEM image observed on the $P$ plane of the composites with $0.5 \% \mathrm{CNF}$ content fabricated via SPS followed by hot extrusion. For observation, the surface was polished and etched using nitric acid solution. The CNFs are confirmed to be oriented in the extrusion direction. The image also clearly shows that the dispersion of CNFs is incomplete, as evidenced by the observed bundle of CNFs, although agglomeration of CNFs was not identified by the SEM observation of inside mixed powder (Fig. 2).

In Fig. 6, thermal conductivity perpendicular to the $P$ plane in the specimen with $2.0 \% \mathrm{CNFs}$ is higher than that perpendicular to the $T$ plane. This difference is due to

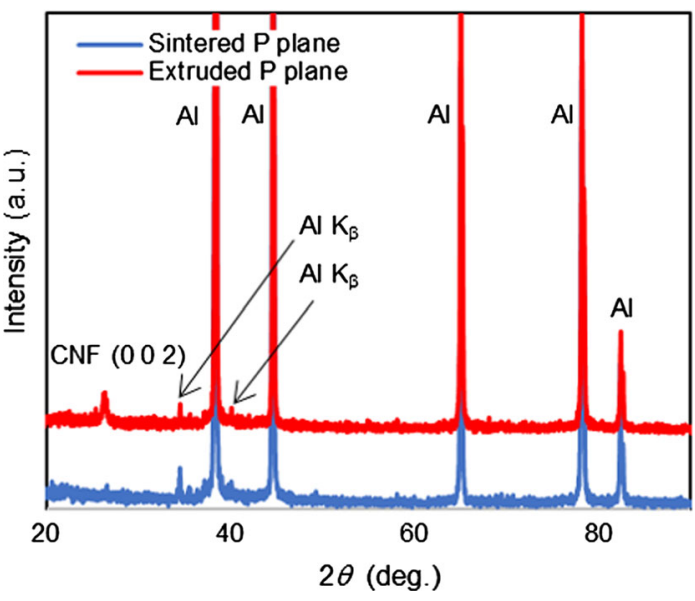

Fig. 7 XRD patterns of $4.0 \% \mathrm{CNF}$-aluminum composites fabricated via extrusion followed by sintering

agglomeration and incomplete alignment of CNFs. By contrast, the thermal conductivity perpendicular to the $T$ plane at $4.0 \% \mathrm{CNF}$ increases, because of the smaller powder particle size.

Figure 9 shows the thermal conductivity of extruded composites fabricated via powder extrusion with R9. The thermal conductivities of composites fabricated via SPS and SPS followed by hot extrusion are also shown. The thermal conductivities of the composites fabricated via powder extrusion monotonically decrease with increasing volume fraction of CNFs.

This decrease in thermal conductivity is attributed to imperfect alignment of CNFs and interfacial thermal resistance. The thermal conductivities of the samples fabricated via powder extrusion are higher than those of the composites fabricated via SPS and SPS followed by hot extrusion, except for the sample with a CNF volume fraction of $4 \%$. The high thermal conductivities are attributed to good dispersion and alignment of the CNFs. 


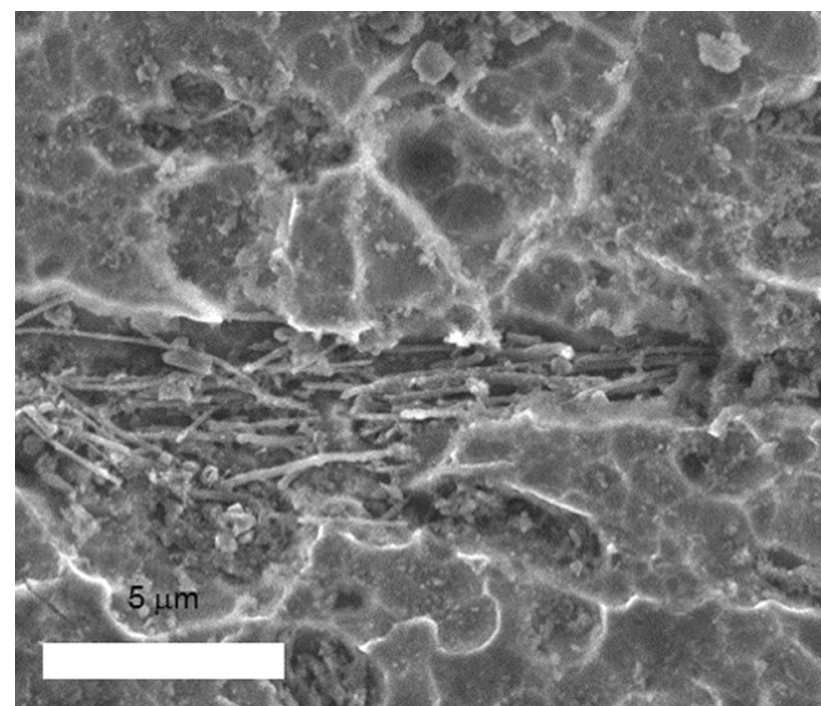

Fig. 8 SEM image obtained on the $P$ plane of the $0.5 \% \mathrm{CNF}-$ aluminum composite fabricated by extrusion after sintering



Fig. 9 Thermal conductivities of composites fabricated by powder extrusion, SPS, and hot extrusion in conjunction with SPS

Low-intensity peaks of $\mathrm{Al}_{4} \mathrm{C}_{3}$ are observed in the XRD patterns of composites fabricated via powder extrusion [24]. This $\mathrm{Al}_{4} \mathrm{C}_{3}$ is mainly formed by the fracture of CNFs during milling. However, SEM observations of the etched surface of the composites confirm that CNFs maintain their shape and no CNFs converted into $\mathrm{Al}_{4} \mathrm{C}_{3}$ rod [25]. The reaction between $\mathrm{CNFs}$ and the aluminum matrix is limited to the interface; its effect on thermal conductivity is negligible. At a CNF volume fraction of $4 \%$, the thermal conductivity of the composite fabricated via SPS followed by extrusion is higher than that of the composite fabricated by powder extrusion. These results suggest that percolation of the CNFs increased the thermal conductivity of the composites because of the mild dispersion state compared with the lower thermal conductivity that originated from the homogeneously dispersed state of CNFs (Fig. 10).



Fig. 10 SEM image of a 1.0\% CNF-reinforced composite fabricated by powder extrusion

The composites at CNF fractions of $0.5 \%$ and $1.0 \%$ fabricated via SPS without extrusion had higher thermal conductivity than the pure aluminum materials (Fig. 4). In contrast, the thermal conductivities of the extruded composites were not improved compared to those of the aluminum materials. This lack of improvement is probably due to the deleterious effects of hot extrusion, namely the shortening of CNFs and a large strain in aluminum matrix. In the extruded composites, CNFs shorter than $2 \mu \mathrm{m}$ were identified. Shortening reinforcements degrades the overall thermal conductivities of composites [27], which is the main cause of the lower thermal conductivity compared to that of pure aluminum materials.

\subsection{Tensile Properties}

Figure 11 shows the nominal stress-nominal strain curve of the $0.5 \% \mathrm{CNF}$-aluminum composite fabricated via SPS followed by hot extrusion. The tensile strengths of the composite and pure aluminum are 146.8 and $137.5 \mathrm{MPa}$, respectively. Thus, a slight increase in tensile strength is observed compared with that of pure aluminum. This improvement is mainly due to load bearing by the CNFs because almost no grain refinement is induced during ball milling.

Figure 12a shows dimples formed around fractured CNFs. The average size of the dimples is $5 \mu \mathrm{m}$. However, the higher-magnification image in Fig. $12 \mathrm{~b}$ shows agglomerated CNFs and cracks generated around them. Thus, the agglomeration of CNFs lowered the strength of the composites. The tensile strength of $4.0 \%$ CNF-reinforced composite was evaluated to be $102.4 \mathrm{MPa}$, which is lower than that of the pure aluminum specimen. The agglomeration of CNFs lowered the tensile strength, 


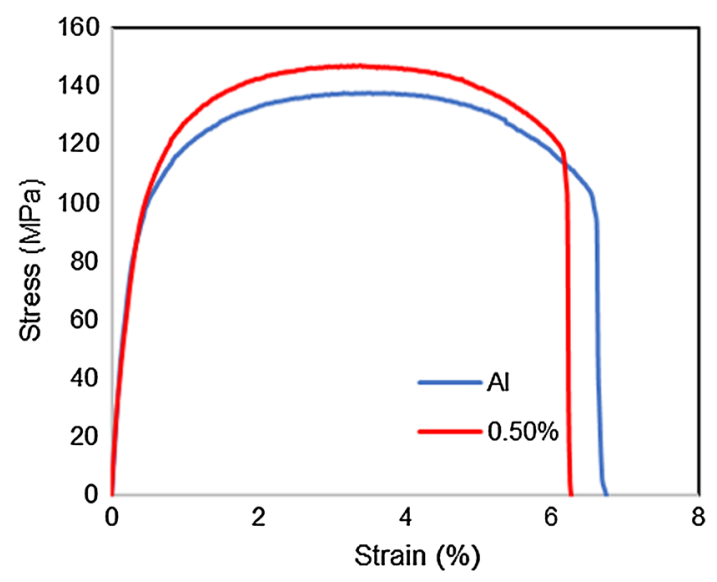

Fig. 11 Nominal stress-nominal strain curve of the $0.5 \% \mathrm{CNF}-$ aluminum composite fabricated by SPS and hot extrusion; the corresponding curve for pure aluminum is also shown

irrespective of the high thermal conductivity of composites. The tensile strengths of composites fabricated via SPS and hot extrusion were lower than values reported in the literature $[10,11,13-15]$ because of the mild dispersion conditions of CNFs and their poor dispersion.

Figure 13 shows the true stress-true strain curves of composites fabricated via powder extrusion with R9. True strain and true stress were calculated by load and stroke using Segal's theory [28]. The tensile strength of the composites fabricated via powder extrusion was greater than those fabricated via SPS followed by hot extrusion. This greater tensile strength of the composites fabricated by powder extrusion is attributed to aluminum grain refinement due to ball milling.

The average grain size of the $0.5 \% \mathrm{CNF}$-reinforced composites is $265.8 \mathrm{~nm}$ [24]. The fracture strains of the composites with $0.5 \%$ and $1.0 \% \mathrm{CNF}$ contents are higher than that of aluminum because of the refinement and recovery of the aluminum grains surrounding CNFs [24].
Figure 14a shows the fracture surface of the $0.5 \% \mathrm{CNF}$ reinforced composites. Dimples are formed around fractured CNFs. The average size of the dimples is $\sim 1 \mu \mathrm{m}$, which is substantially smaller than that of composites fabricated via SPS followed by hot extrusion. This smaller dimple size is attributed to refined aluminum grains and good dispersion of the CNFs. However, Fig. 14b shows brittle fracture around clustered aluminum, indicating fractured aluminum particles marked by white arrows. This area corresponds to the region in which small aluminum grains are clustered, as denoted by white circles in Fig. 15, a field-emission scanning electron microscopy (FESEM) image of aluminum grains in the $0.5 \% \mathrm{CNF}$-aluminum composites fabricated via powder extrusion at $\mathrm{R}$.

These clustered regions should be useful for improving the thermal conductivity of composites. However, they also deteriorate the fracture strain of the composites.

Figure 16 shows the true stress-true strain curves of composites fabricated by powder extrusion with R16. The fracture strain of the aluminum fabricated by powder extrusion with R16 is higher than that of the aluminum fabricated by powder extrusion with R9 (Fig. 13). This result is attributed to differences in the aluminum grain structures of the samples.

Figure 17 shows a comparison of the fracture surfaces of the aluminum materials fabricated by powder extrusion with different extrusion ratios. Numerous fine dimples are observed in the sample extruded with R16, whereas fractured hard particles of aluminum are observed, as indicated by white arrows in Fig. 17b. These aluminum hard particles correspond to regions comprising small aluminum grains, as denoted by white circles in Fig. 15. Spatial distribution in hardness due to variability in aluminum grain size exists and the region with high hardness bears a large load. Fracture of this region decreased the fracture strain of the aluminum. A longer holding duration at $550{ }^{\circ} \mathrm{C}$ during
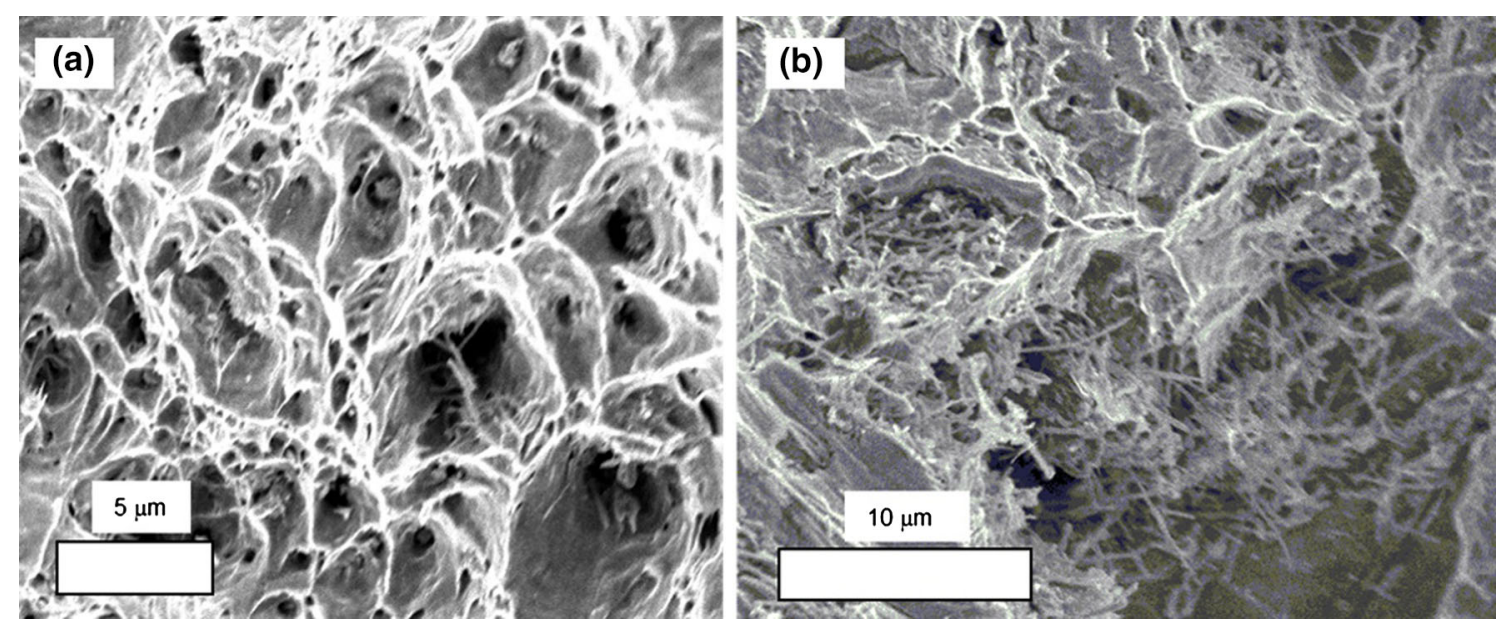

Fig. 12 SEM images of the fracture surface of the $0.5 \% \mathrm{CNF}$-aluminum composite fabricated by SPS and hot extrusion 


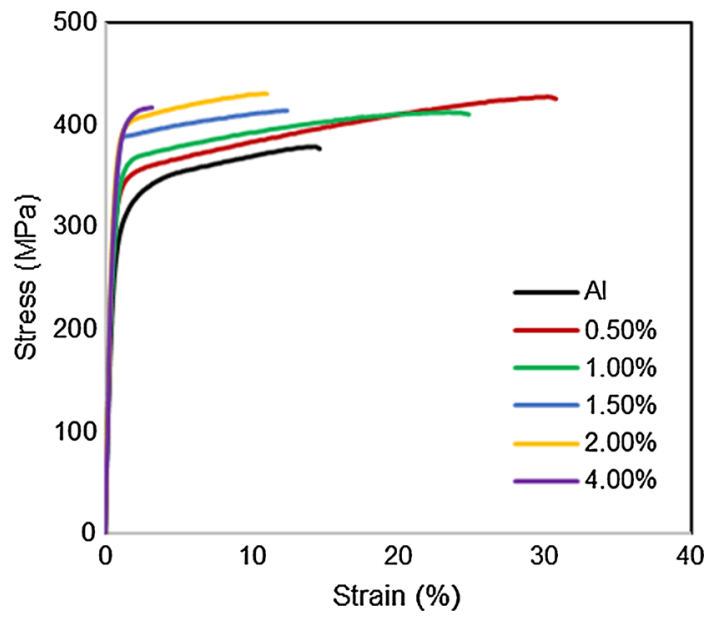

Fig. 13 True stress-true strain curves of composites fabricated by powder extrusion with an extrusion ratio of 9

extrusion at R16 homogenized the microstructure of aluminum, increasing its strength and ductility.

At a CNF volume fraction of $0.5 \%$, fracture strain does not substantially change in the sample extruded at R16 compared with that of the sample extruded at R9. By contrast, at a CNF volume fraction of $2.0 \%$, the fracture strain of the specimen extruded at R16 is greater than that of the specimen extruded at R9. In addition, a comparison of the fracture strains reveals that the fracture strain of the sample with a CNF volume fraction of $5.0 \%$, fabricated with R16 is higher than that of the sample with a CNF fraction of $4.0 \%$ fabricated with R9. Figure 18 compares the true stress-true strain curves of the samples with a CNF fraction of $2.0 \%$, extruded at R9 and R16.

The $0.2 \%$ proof stress of composites fabricated with R 16 is slightly lower than that of composites fabricated with R9 because of larger aluminum grains. However, the region of strain hardening is wider for the sample prepared with R16,

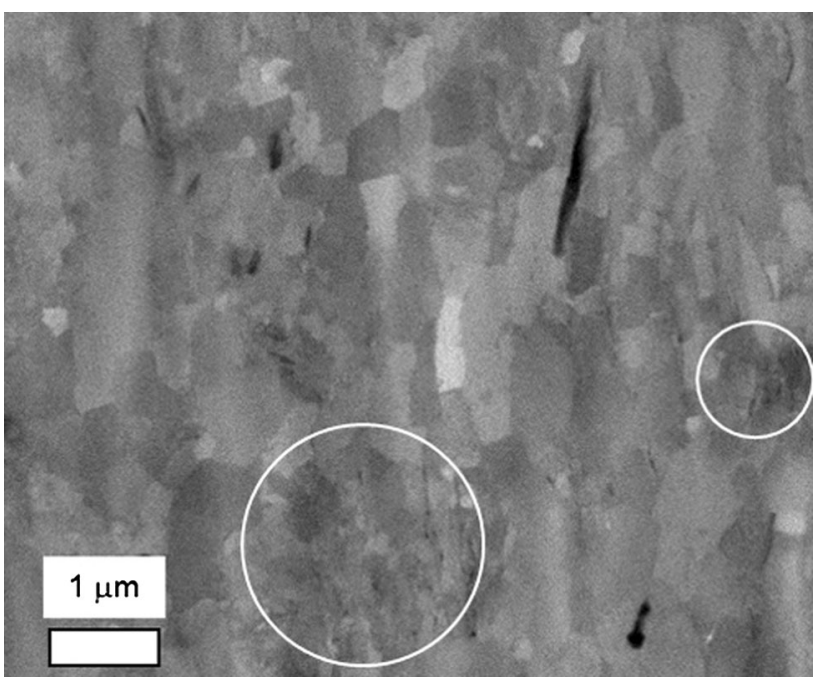

Fig. 15 FESEM image of aluminum grains in $0.5 \% \mathrm{CNF}$-aluminum composites fabricated by powder extrusion at an extrusion ratio of 9

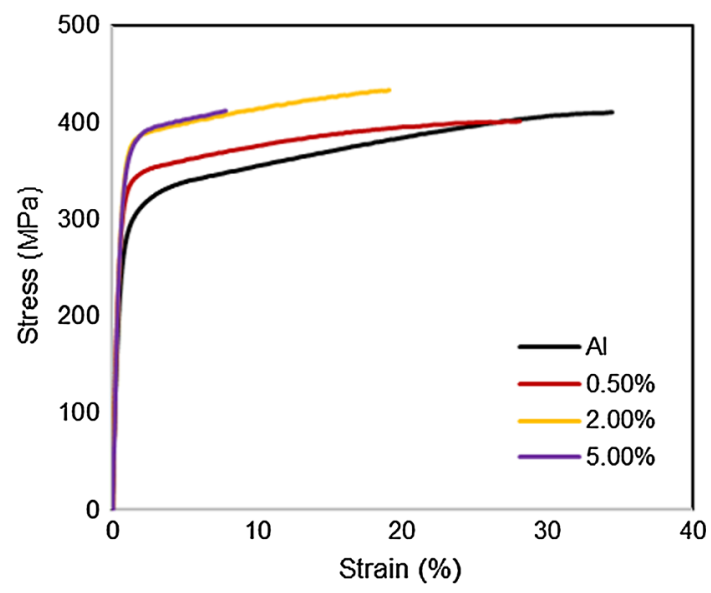

Fig. 16 True stress-true strain curves of composites fabricated by powder extrusion with an extrusion ratio of 16


Fig. 14 Fracture surface of $0.5 \%$ CNF-reinforced composites fabricated by powder extrusion with an extrusion ratio of 9 

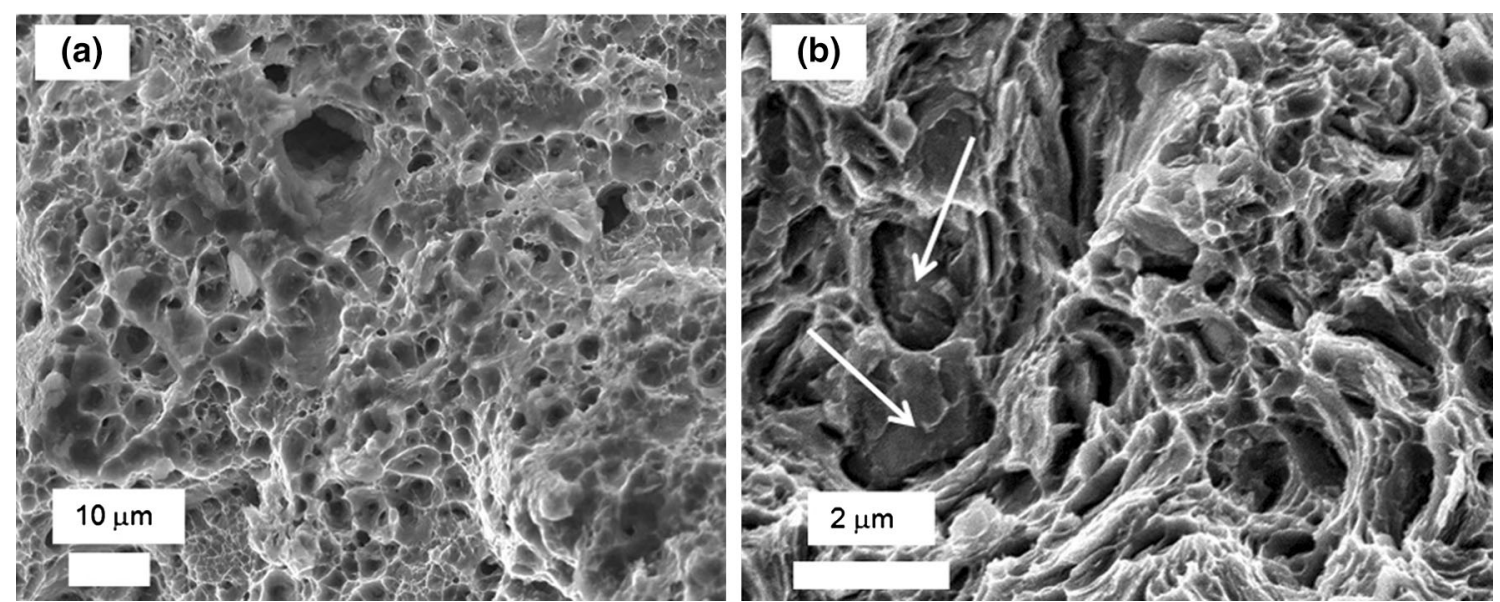

Fig. 17 Fracture surfaces of aluminum materials fabricated by powder extrusion with an extrusion ratio of a 16, b 9

and its tensile strength is approximately the same as that of the sample prepared with R9. The areas under the stressstrain curves correspond to the fracture energy of the composites. The fracture energy of composites prepared with R16 is much larger than that of the composites prepared with R9 likely because of the change in microstructure due to long heating duration and high extrusion ratio.

Figure 19 shows the fracture surfaces of the composite fabricated with R9. In Fig. 19a, dimples appear in the aluminum matrix, as well as fractured aluminum particles (red arrows) and detachment of aluminum particles from aluminum matrix (blue arrows). White arrows indicate cracks, and orange arrows indicate pulled-out CNFs. Figure $19 \mathrm{~b}$ shows a magnified view of the other position around the fractured CNFs. Green arrows indicate fractured $\mathrm{CNFs}$, around which dimples are formed. The orange arrow shows a pulled-out CNF, and black arrows indicate CNFs

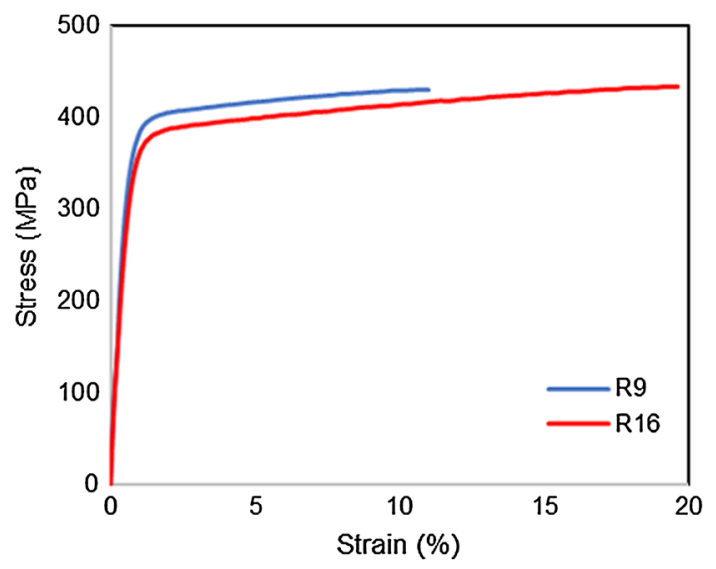

Fig. 18 Comparison of true stress-true strain curves of $2.0 \% \mathrm{CNF}-$ aluminum composites fabricated by powder extrusion with extrusion ratios of 9 and 16 oriented perpendicular to longitudinal direction of the specimen.

Figure 20 shows the aluminum grains in the composite fabricated via powder extrusion with R9; CNFs are denoted in black. CNFs are aligned to some extent, while a few nonaligned CNFs are observed. Average grain size of aluminum is $232.7 \mathrm{~nm}$. Figure 21 shows the FESEM image of the R9 composite at different positions.

The white circles in Fig. 21 indicate regions comprising small aluminum grains, and the region outside the circle comprises larger grains. The fractured aluminum particle in Fig. 19a corresponds to a region comprising small aluminum grains. Black arrows indicate small aluminum grains, whereas red arrows indicate larger grains. Kikuchi et al. [29] studied fatigue crack propagation in harmonicstructured titanium alloys, in which large grains were surrounded by networks of smaller grains. They reported that the cracks propagated along the boundaries between fine and coarse grains. In this study, cracks are assumed to initiate in a region comprising small aluminum grains, such as the region indicated by the circle with the letter $\mathrm{A}$, and to propagate along the orange dashed line, leading to the fracture surface shown in Fig. 19a.

Figure 22a shows fracture surface of the composite fabricated with R16. Many dimples appear on the fracture surface; fracture surface is highly ductile. Figure $22 \mathrm{~b}$ shows a magnified view of Fig. 22a, which exhibits a rougher fracture surface than that in Fig. 19b. Dimples appear around the fractured CNFs. In addition, no CNFs oriented perpendicular to the longitudinal direction of the specimen and no pulled-out CNFs appear. These findings can be attributed to the better orientation and high $\mathrm{CNF} /$ aluminum interfacial strength due to the high extrusion ratio and longer heating duration.

Figure 23 shows aluminum grains in the composite fabricated via powder extrusion with R16. No regions with 



Fig. 19 Fracture surface of $2.0 \% \mathrm{CNF}$-aluminum composites fabricated by powder extrusion with extrusion ratio of 9



Fig. 20 FESEM image of aluminum grains in $2.0 \% \mathrm{CNF}$-aluminum composites fabricated by powder extrusion at extrusion ratio of 9

clustered small aluminum grains appear in Fig. 23, wherein black arrows indicate CNFs. Small aluminum grains are generated around the CNFs by recrystallization, as indicated by white arrows, and the size of the grains increases with increasing distance from the CNFs. Smaller grains are surrounded by larger grains, and the structure exhibits periodicity. This region is indicated by a dashed ellipsoid. Large grains are easily deformed, forming voids. Void growth is inhibited by the load-bearing capacity of the $\mathrm{CNFs}$ and the small aluminum grains.

Figure 24 shows an FESEM image of R16 composites at a different position. The CNFs are clearly well oriented due to the higher extrusion ratio. Grains around the CNFs are small, whereas some CNFs are incorporated into the aluminum grains, as indicated by arrows. In the R16 composites, more CNFs are incorporated into aluminum grains than in the R9 composites. This difference is probably due to the longer heating duration and the incorporation of

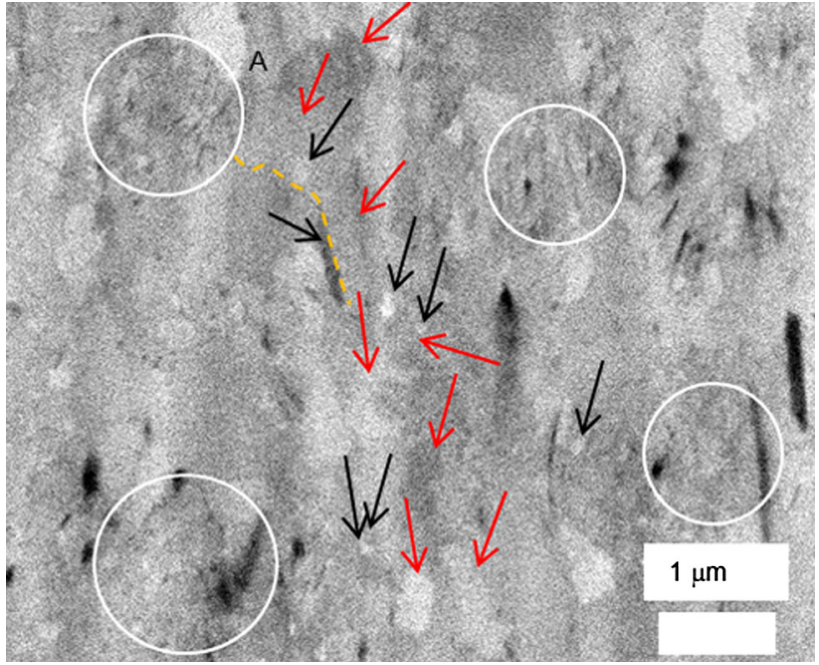

Fig. 21 FESEM image of aluminum grains in 2.0\% CNF-aluminum composites fabricated by powder extrusion at extrusion ratio of 9 at a different position from Fig. 20

CNFs into aluminum grains during heating before extrusion. The incorporation of CNFs into aluminum grains leads to the high ductility of the composites because of the strong interfacial interaction between CNFs and aluminum.

SEM observations of the etched surface of R16 composites revealed that CNFs shorter than $1 \mu \mathrm{m}$ in composites [30], which are shorter than the shortest CNFs in R9 composites. A large extrusion ratio resulted in fractured CNFs. However, the incorporation of CNFs into aluminum grains improved the interfacial strength and the shortening of CNFs did not decrease the tensile strength or elongation of R16 composites.

Wang et al. [31] reported that the so-called heterogeneous grain structure leads to high strength and ductility. In this structure, a mixture of small and large grains and their varied sizes contribute to high strength and ductility. In this study, 

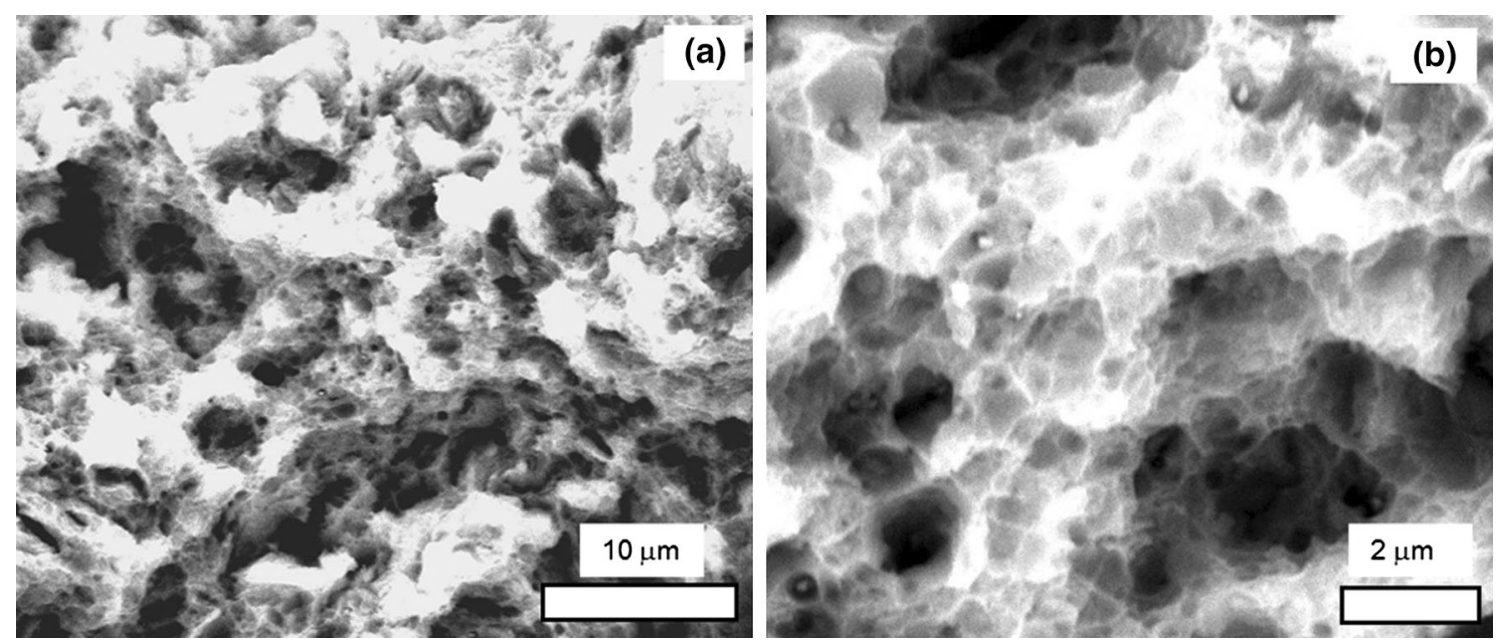

Fig. 22 Fracture surface of 2.0\% CNF-aluminum composites fabricated by powder extrusion with an extrusion ratio of 16

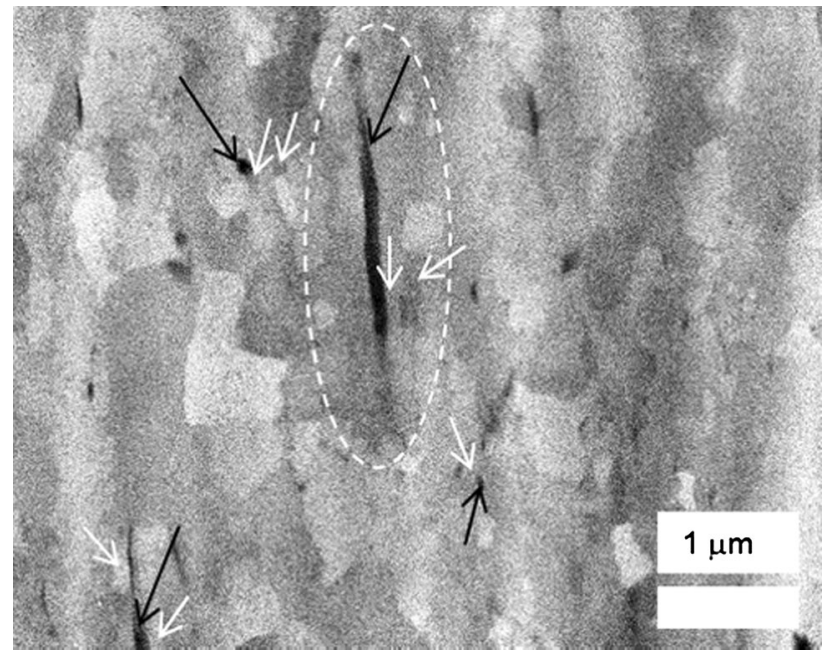

Fig. 23 FESEM image of aluminum grains in 2.0\% CNF-aluminum composites fabricated by powder extrusion at an extrusion ratio of 16

the size variation and spatial arrangement of aluminum grains contributed to high ductility, whereas larger grains than those in the composite fabricated via powder extrusion with $\mathrm{R} 9$ resulted in a lower $0.2 \%$ proof stress. Importantly, the load-bearing capacity of CNFs delays void growth originating from the deformation of large aluminum grains.

This study clearly elucidated the fracture process of our composites. For $2.0 \%$ CNF-reinforced composites fabricated via powder extrusion with $\mathrm{R} 9$, cracks were initiated at fractured aluminum particles and propagated along the boundaries between small and large grains, leading to particle detachment. Imperfectly aligned CNFs and CNFs pulled out from the aluminum matrix also caused fracture. For 2.0\% CNF-reinforced composites fabricated via powder extrusion with R16, better alignment of CNFs and a stronger CNF/aluminum interface improved the tensile properties. The clustered regions of small aluminum grains

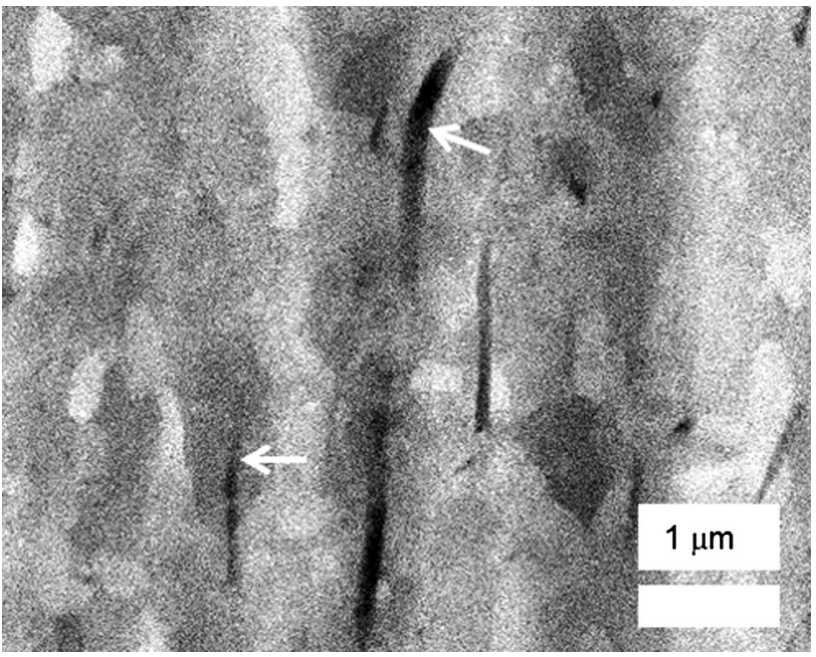

Fig. 24 FESEM image of aluminum grains in $2.0 \% \mathrm{CNF}$-aluminum composites fabricated by powder extrusion at an extrusion ratio of 16 in a different position from Fig. 23

and the grain structure, in which recrystallization occurred around CNFs, were eliminated, and the grain size was increased with increasing distance from CNFs to improve the ductility of composites, irrespective of the decreased $0.2 \%$ proof stress. CNFs incorporated into aluminum grains also increased the ductility, which contradicts the wellknown fact that fracture strain decreases at a high extrusion ratio due to work hardening, while fabricated composites are denser. This study also elucidated a strategy to fabricate composites with superior properties and clearly demonstrated that well-dispersed CNFs and a moderate reaction at the $\mathrm{CNF} /$ aluminum interface lead to high thermal conductivity. Further, the percolation of CNFs at a high CNF fraction without $\mathrm{Al}_{4} \mathrm{C}_{3}$ formation further increases thermal conductivity. Regarding the tensile strength and ductility, a longer heating duration and high extrusion ratio led to high 
ductility and thus high fracture energy. The fabrication method used herein simply combined conventional ball milling and encapsulating powders into aluminum containers in vacuum. At first glance, the method did not seem particularly new; however, the milling conditions were carefully determined to homogeneously disperse the CNFs and to prevent CNFs from fracturing during milling. However, recrystallization, grain growth, and the elimination of clustered aluminum grains, which together resulted in a unique structure, led highly ductile composites. Thus, appropriately controlling the microstructure by optimizing the fabrication process may realize CNF-reinforced aluminum-matrix composites with superior properties.

\section{Conclusions}

1. Anisotropy exists even in the thermal conductivities of sintered $\mathrm{CNF}$-aluminum composites. The thermal conductivity in the radial direction is higher than that in the press direction.

2. The thermal conductivity is controlled via hot extrusion. The longitudinal conductivity is higher than the transverse conductivity.

3. The thermal conductivity of the composites fabricated via powder extrusion is higher than that of the composites fabricated via SPS followed by hot extrusion, except in the case of a CNF volume fraction of $4.0 \%$. This result is attributed to dense structure and alignment of the CNFs. The percolation of CNFs leads to greater conductivity in composites with $4.0 \%$ CNFs fabricated via SPS followed by hot extrusion, whereas tensile strength is lower than that of pure aluminum.

4. Tensile strength and ductility largely vary with the ball-milling conditions of the $\mathrm{CNF}$ and aluminum mixtures and with the hot extrusion conditions. The tensile strength of the composites fabricated via mild milling and SPS followed by extrusion is the lowest because of almost no grain refinement. The composites fabricated via powder extrusion with R9 exhibit the highest tensile strengths among the composites fabricated in the present study because of grain refinement. The fracture strains of the composites fabricated by powder extrusion with R16 are higher than those of the composites fabricated with R9 when the CNF volume fraction is $2 \%$ or $5 \%$. The results demonstrate that appropriately controlling the heating and plastic deformation during extrusion is important for fabricating composites with high ductility.

Acknowledgements This work was partly supported by a Grant-inAid for Scientific Research from the Ministry of Education, Culture, Sports, Science and Technology, Japan. Fumio Ogawa would like to express his sincere gratitude to the Mitsubishi Material Corporation for their financial support. Financial supports from the Light Metal Educational Foundation Inc. and the Japan Aluminium Association are also greatly acknowledged. Dr. Toshiyuki Nishimura of the National Institute for Materials Science is acknowledged for his support in the measurement of thermal conductivity of composites.

\section{References}

[1] S. Berber, Y.K. Kwon, D. Tománek, Phys. Rev. Lett. 84, 4613 (2000)

[2] B.G. Demczyk, Y.M. Wang, J. Cumings, M. Hetman, W. Han, A. Zettl, R.O. Ritchie, Mater. Sci. Eng. A 334, 173 (2002)

[3] M.F. Yu, O. Lourie, K. Moloni, T.F. Kelly, R.S. Ruoff, Science 287, 637 (2000)

[4] M.F.L. De Volder, S.H. Tawfick, R.H. Baughman, A.J. Hart, Science 39, 535 (2013)

[5] S. Suresh, A. Mortensen, A. Needleman, Fundamentals of Metal Matrix Composites (Butterworth-Heinemann, Oxford, 1993)

[6] D.B. Miracle, Compos. Sci. Technol. 65, 2526 (2005)

[7] K.U. Kainer, Metal Matrix Composites. Custom-Made Materials for Automotive and Aerospace Engineering (Wiley, Weinheim, 2006)

[8] R. George, K.T. Kashyap, R. Rahul, S. Yamdagnim, Scr. Mater. 53, 1159 (2005)

[9] H. Uozumi, K. Kobayashi, C. Masuda, M. Yoshida, Adv. Mater. Res. 15-17, 209 (2006)

[10] C. He, N. Zhao, C. Shi, X. Du, J. Li, H. Li, Q. Cui, Adv. Mater. 19, 1128 (2007)

[11] H.J. Choi, G.B. Kwon, G.Y. Lee, D.H. Bae, Scr. Mater. 59, 360 (2008)

[12] T. Laha, Y. Chen, D. Lahiri, A. Agarwal, Compos. Part A 40, 589 (2009)

[13] H. Choi, J. Shin, B. Min, J. Park, D. Bae, J. Mater. Res. 24, 2610 (2009)

[14] A.M.K. Esawi, K. Morsi, A. Sayed, A.A. Gawad, P. Borah, Mater. Sci. Eng. A 508, 167 (2009)

[15] A.M.K. Esawi, K. Morsi, A. Sayed, M. Taher, S. Lanka, Compos. Sci. Technol. 70, 2237 (2010)

[16] H. Kwon, D.H. Park, J.F. Silvain, A. Kawasaki, Compos. Sci. Technol. 70, 546 (2010)

[17] S.R. Bakshi, A. Agarwal, Carbon 49, 533 (2011)

[18] H. Kwon, H. Kurita, M. Leparoux, A. Kawasaki, J. Nanosci. Nanotechnol. 11, 4119 (2011)

[19] L. Jiang, Z. Li, G. Fan, L. Cao, D. Zhang, Scr. Mater. 66, 331 (2012)

[20] S.E. Shin, H.J. Choi, D.H. Bae, J. Compos. Mater. 47, 2249 (2012)

[21] J. Wu, H. Zhang, Y. Zhang, X. Wang, Mater. Des. 41, 344 (2012)

[22] B. Chen, S. Li, H. Imai, L. Jia, J. Umeda, M. Takahashi, K. Kondoh, Mater. Des. 72, 1 (2015)

[23] H. Kwon, M. Leparoux, Nanotechnology 23, 415701 (2012)

[24] F. Ogawa, C. Masuda, Compos. Part A 71, 84 (2015)

[25] F. Ogawa, S. Yamamoto, C. Masuda, Mater. Sci. Eng. A 711, $460(2018)$

[26] F. Ogawa, C. Masuda, H. Fujii, J. Mater. Sci. 53, 5036 (2018)

[27] C.W. Nan, R. Birringer, D.R. Clarke, H. Gleiter, J. Appl. Phys. 81, 6692 (1997)

[28] V.M. Segal, S. Ferrasse, F. Alford, Mater. Sci. Eng. A 422, 321 (2006)

[29] S. Kikuchi, T. Imai, H. Kubozono, Y. Nakai, M. Ota, A. Ueno, K. Ameyama, Int. J. Fatigue 92, 616 (2016)

[30] F. Ogawa, C. Masuda, J. JILM 63, 350 (2013)

[31] Y. Wang, M. Chen, F. Zhou, E. Ma, Lett. Nat. 419, 912 (2002) 